Hernández De la Torre, E. \& González Miguel, S. (2020). Análisis de datos cualitativos a través del sistema de tablas y matrices en investigación educativa. Revista Electrónica Interuniversitaria de Formación del Profesorado, 23(3), 115-132.

DOI: https://doi.org/10.6018/reifop.435021

\title{
Análisis de datos cualitativos a través del sistema de tablas y matrices en investigación educativa
}

\author{
Elena Hernández De la Torre, Sandra González Miguel \\ Universidad de Sevilla
}

\section{Resumen}

Este artículo tiene como objetivo aportar sistemas de análisis cualitativo de los datos generados a partir de la información obtenida en entrevistas de grupos focales y grupos de discusión como método de aprendizaje compartido y con carácter conversacional. Se han llevado a cabo entrevistas con dos grupos de expertos en educación, por una parte grupos focales formados por $4 / 5$ profesores-asesores de centros de profesorado; por otro lado grupos de discusión formados por 4/6 profesores-mentores. Se ha utilizado un grupo de cada estrategia grupal para el análisis cualitativo de los datos, siendo los tópicos utilizados en los grupos focales "Ventajas e inconvenientes de la participación de centros educativos en redes escolares" y en los grupos de discusión "Identificación de obstáculos y barreras en el proceso de asesoramiento a docentes principiantes". En ambos casos se ha profundizado en la conversación sobre sus propias experiencias profesionales siendo analizadas con sistemas de tablas y matrices de las cuales proponemos dos modelos. Se concluye la necesidad de utilizar modelos rigurosos de análisis sistemático de la información obtenida con el objetivo de construir teorías sólidas por parte de los entrevistados sobre los tópicos a tratar en experiencias compartidas para la construcción del conocimiento.

\section{Palabras clave}

Análisis de datos cualitativos; entrevistas grupales; matrices; tablas. 


\title{
Analysis of qualitative data through the system of tables and matrices in educational research
}

\begin{abstract}
This article aims to provide qualitative analysis systems of the data generated from the information obtained in interviews of focus groups and discussion groups as a method of shared and conversational learning. Interviews have been carried out with two groups of experts in education, on the one hand focus groups formed by $4 / 5$ teachers-consultants of teaching centers; on the other hand discussion groups formed by $4 / 6$ teacher-mentors. A group of each group strategy has been used for the qualitative analysis of the data, the topics being used in the focus groups "Advantages and disadvantages of the participation of schools in school networks" and in the discussion groups "Identification of obstacles and barriers in the process of advising beginner teachers". In both cases, the conversation about their own professional experiences has been deepened, being analyzed with systems of tables and matrices of which we propose two models. It concludes the need to use rigorous models of systematic analysis of the information obtained with the objective of building solid theories by the interviewees on the topics to be treated in shared experiences for the construction of knowledge.
\end{abstract}

\section{Key words}

Qualitative data analysis; group interviews; matrixes; tables.

\section{Introducción}

En la investigación educativa actual, la utilización de estrategias grupales versus individuales para la obtención de datos es cada vez más frecuente. Se trata de una forma de recolectar datos cualitativos en grupos de personas dispuestas a conversar acerca de un tópico o tema específico. Si bien es cierto que las personas dialogan en grupo de forma más cómoda generando numerosas ideas en este ambiente distendido e intercambiando experiencias, la cantidad de datos que se producen requiere de un análisis minucioso en estos diálogos, existiendo escasas directrices acerca de cómo examinar los datos que se originan a partir del análisis de contenido. Entre las opciones para organizar este análisis se encuentra la utilización de sistemas de tablas/matrices para ordenar y clasificar la información cualitativa obtenida.

En este artículo se encuentra la presentación de un marco de análisis de datos cualitativos para la investigación en educación a partir del uso de sistemas de matrices y tablas, con la función principal de ordenar el contenido que representa la información obtenida en función de los códigos y objetivos planificados. Adentrarse en la temática significa definir que los grupos focales y los grupos de discusión son estrategias de investigación cualitativa que generan datos que requieren un microanálisis de la conversación verbal del grupo, con lo que habría que aumentar la rigurosidad, tal y como señalan Onwuegbuzie, Dickinson, Leech y Zoran (2011), el "rigor de los análisis de los grupos participantes" (p. 127).

\section{Eficacia/empleo de los grupos focales y grupos de discusión para la investigación cualitativa en educación}


En la actualidad los grupos focales y grupos de discusión son consideradas técnicas de recolección de datos interesantes y valiosos a través de la conversación, ya que se obtiene información acerca de las opiniones de un grupo de profesionales para indagar sobre la percepción de las personas en torno a un tema en particular (Wilkinson, 2004; 2012). Se fundamentan en una epistemología cualitativa, con carácter práctico y explicativo del conocimiento, y basado en una construcción "que se genera al confrontar el pensamiento del investigador con los múltiples eventos empíricos que se presentan" (Hamui-Sutton y Varela-Ruiz, 2013, p. 56).

Sus principios provienen, por una parte de la Psicología Clínica, la cual indica que las personas escuchan, hablan y se comunican con mayor facilidad encontrándose en grupos conversacionales (Viñas, 2008), y por otra, de la década de los 30's donde los investigadores sociales "diseñaron estrategias grupales que permitieran más libertad y apertura a los entrevistados" (Hamui-Sutton y Varela-Ruiz, 2013, p. 56). Hoy en día se usan estas técnicas mediante los recursos tecnológicos de videoconferencias (Skype, Blackboard, etc.) y grupos de internet (WhatsApp, foros, chats, etc.), por lo que se trata de modelos comprensivos de la construcción del conocimiento basados en un proceso dialógico de análisis del discurso acerca de los problemas sociales. Atendiendo al ámbito educativo, se detecta el uso de la teoría fundamentada para el análisis de los datos "tratando de descubrir y explicar, mediante una metodología inductiva, la interpretación de significados desde la realidad social de los individuos, con el fin último de crear una teoría que explique el fenómeno de estudio" (Vivar, Arantzamendi, López-Dicastillo y Gordo (2010, p. 1). En ambos casos se trata de entrevistas grupales en las que todos los participantes entrevistados se encuentran citados en el mismo lugar y hora compartiendo espacio de diálogo, conversación y socialización en respuesta a una serie de cuestiones planteadas por el investigador y siguiendo la misma temática o varias en función de los intereses de la investigación.

Llegados a este punto, se define el grupo focal como entrevista grupal que cuenta con una temática específica y preguntas de investigación justificadas "cuyo sello característico es el uso explícito de la interacción, las actitudes y los puntos de vista sobre un determinado fenómeno; no se desarrolla aisladamente, sino en interacción con otras personas" (García Calvente y Mateo, 2000, p. 181). Asimismo, se define como "un espacio de opinión para captar el sentir, pensar y vivir de los individuos, provocando auto explicaciones para obtener datos cualitativos" (Hamui-Sutton y Varela-Ruiz, 2013, p. 56). Parte de un interés común, de un ambiente relajado y no directivo para comprender cómo entienden y construyen las personas que pertenecen a un grupo los conocimientos, biografías o hechos, sin tener que llegar necesariamente a acuerdos, "importa tanto lo que hay de común como lo que hay de diferente en las experiencias de los participantes" (García Calvente y Mateo, 2000, p. 181).

Respecto a la definición de grupo de discusión, en este instrumento de investigación cualitativa se explora a un grupo de personas que representan a un grupo social en una situación de diálogo y que desarrollan una conversación acerca de un tópico. Barbour, Amo y Blanco (2013) afirman que primero es conveniente realizar entrevistas individuales y posteriormente las grupales, ya que habrá participantes que necesiten contar más secuencias y compartirlas con sus iguales. De igual modo, se deben tener en cuenta que para planificar un grupo de discusión es necesaria una sala donde sólo se encuentre el grupo y el investigador, utilizar un buen equipo de grabación que recoja opiniones y debate, transcribir de forma literal para no perder fragmentos relevantes y tomar notas (impresiones, gestos, comentarios relevantes, etc.).

Entre las ventajas que ofrecen estos métodos en educación está la interacción entre los participantes, la posibilidad de crear ideas compartidas, cooperativas y consensuadas o discrepantes y diferentes en grupo para el estudio de actitudes y experiencias, se trata de 
analizar cómo se desarrollan y utilizan las ideas y cómo se construyen. Asimismo "facilitan la discusión y activan a los participantes a comentar y opinar aún en aquellos temas que se consideran como tabú" (Hamui-Sutton y Varela-Ruiz, 2013, p.56).

Lo común de ambas técnicas es que se llevan a cabo en una habitación amplia y agradable, que debe contar con todas las comodidades de climatización y confort para favorecer la conversación de los participantes, entre 4 y 10 personas, que hablan alrededor de 102 horas. Es necesario buscar personas que tengan determinada experiencia, interés en el tópico de conversación y que sean hombres y mujeres, entre otras variables.

Para poder obtener información segura, deben ser grabadas y observadas las conversaciones en su totalidad, además de una persona encargada de guiar la conversación para que se alcance el objetivo del tópico a tratar, realizando preguntas u observaciones que ayuden a la reflexión del tema que se investiga. La estrategia se basa en reunir al grupo de expertos en la materia, con un determinado perfil, para explorar conocimientos, juicios, razonamientos, limitaciones de un tema determinado. El moderador/a estimula al grupo para la obtención de respuestas, anticipándose a la obtención de ellas, llevando a conversar al grupo, actuando como iniciador del discurso y consensuando conclusiones. Los objetivos principales de ambas técnicas son los siguientes (Ibarrola-García, 2012):

- Recoger información cualitativa sobre deseos, necesidades, conceptos, etc.

- Recoger información rica y variada en poco tiempo.

- Complementar la información recogida mediante otras técnicas.

- Recoger información cualitativa con detalle y gran flexibilidad.

La diferencia entre grupos de discusión y grupos focales (Gutiérrez, 2009) se muestra en la Tabla 1:

Tabla 1.

Grupos de discusión versus grupos focales (citado por Gutiérrez, 2009)

$\begin{array}{ll}G D=\text { grupo de discusión } & F G=\text { focus group/Grupo Focal } \\ \begin{array}{l}\text { Escenario y situación más espontánea y y } \\ \text { natural. }\end{array} & \begin{array}{l}\text { La denominación FG Se usa más en el } \\ \text { ámbito anglosajón }\end{array}\end{array}$

ABIERTO y FLEXIBLE

Apertura Campo de Observación

El moderador va por detrás

$G D$ ofrece antes repuestas al moderador para que éste estimule el discurso grupal.
FOCALIZADO a aspectos concretos de los objetivos de la Investigación Social.

El moderador va por delante

El moderador estimula al FG para la obtención de respuestas discursivas del grupo.
DEJA HABLAR AL GRUPO

Se abren espacios para el habla.
HACE HABLAR AL GRUPO

Finalidad económica e ideológica 
El GD parte del disenso del grupo para luego Grupo consensuado inicialmente llegar a un consenso más tarde.

\section{CONDUCIÓN POCO DIRECTIVA}

El GD tiene una Fase Inicial inmediata a la presentación del grupo y una Fase de Consolidación del Grupo, posicionamiento de los participantes frente al moderador.

\section{CONDUCIÓN DIRECTIVA}

Se prevé una serie de preguntas para focalizar la puesta en común

Como se puede observar (Tabla 1), los grupos focales se centran en aspectos concretos de la investigación social donde el moderador hace hablar al grupo, con el consenso inicial del mismo, siendo la conducción de la misma directiva con una relación expresa de aspectos a debatir. En el caso de los grupos de discusión se aborda de manera informal y en este caso no es focalizada, sino abierta y flexible, lo que crea espacios para el diálogo llegando a un consenso posterior. Estas estrategias de investigación se utilizan normalmente para recabar información junto a otras estrategias de indagación como es la entrevista por las siguientes razones:

- Ofrecen información previa y de primera mano sobre un tema en concreto,

- Se utilizan para explorar este tema de forma más específica y/o general, según la importancia que le confiere el grupo focal, como por ejemplo la mejora del funcionamiento de estas redes, la participación, las dinámicas de trabajo, etc.

- Recaban información consensuada y motivada respecto a puntos en común o divergentes, en su caso,

- Ayudan a desarrollar y construir ideas y teorías acerca de los tópicos que se tratan en estas redes, ya que, al llevar a la reflexión conjunta, los participantes pueden desarrollar conceptos y formas de trabajo,

- Ayudan a elaborar ideas o enfoques diferentes a los preestablecidos en investigaciones anteriores acerca de este tema, rebatiendo las ideas iniciales planteadas.

\section{Sistemas de representación de datos cualitativos a partir de entrevistas}

El análisis de los datos en investigación cualitativa a partir de grupos focales y grupos de discusión es un tema de vital importancia para representar la información de forma objetiva y viable. Representar los resultados de forma gráfica facilita la credibilidad de esos resultados y la toma de decisiones respecto a las conclusiones. De este modo, es necesario en la investigación cualitativa optar por un modelo de representación de datos eficaz para codificar la ingente cantidad de datos y no convertir su lectura en una sucesión de declaraciones.

Este artículo no se refiere a matrices utilizadas en investigación que tienen como objetivo "mostrar cualquier tipo de idea y especialmente procesos y relaciones de influencia" (Codina, 2019, p.1), más bien se ajusta al uso de Tablas y Matrices que permitan visibilizar y sintetizar la información, no tratándose de registros en una hoja de cálculo, sino en sintetizar 
resultados de investigaciones cualitativas al igual que se realiza en el caso de investigaciones de tipo cuantitativo.

Existen una serie de reglas para componer las Tablas. Una tabla o matriz se compone de “...una serie de filas y columnas. El cruce de las mismas produce celdas que contienen valores. Esta simple estructura es una poderosa forma de sintetizar información y de mostrar relaciones, ya se trate de datos cualitativos o cuantitativos" (Codina, 2019, p. 1). La forma usual de representación son las Tablas horizontales y verticales, como se muestran en estos ejemplos (Codina, 2019; Miles y Huberman, 2004; Miles, Huberman, y Saldaña, 2014; Onwuegbuzie et al, 2011):

- Tablas como fichas (citado por Codina, 2019):

Tabla 2.

Ejemplo 1 de tabla como ficha

\begin{tabular}{l}
\hline Nombre \\
\hline Fecha de creación \\
País de origen \\
Cobertura geográfica \\
Cobertura temática
\end{tabular}

Se destaca en la fila superior denominada "nombre" la entidad principal de estudio y en las filas continuas encontramos una serie de aspectos concretos que se van ampliando en las columnas creadas a la derecha.

Tabla 3.

Ejemplo 2 de tabla como ficha

$\begin{array}{ll}\text { N. Observaciones } & \text { Respuesta } \\ \text { Evaluador } & \text { Autores }\end{array}$

1

2

En este ejemplo de Tabla, se añaden tres columnas en la parte superior en la que la primera de ellas denominada "N" se refiere al aspecto general o código a analizar y en las columnas siguientes nos indican que se debe atender a los datos de las observaciones por parte del evaluador, entrevistador, asesor, etc., y la tercera columna es para las respuestas de los participantes o autores. A su vez, en esta ficha se pueden incorporar infinidad de datos numerados consecutivamente en las diferentes filas representadas. En este punto se muestran dos ejemplos de las llamadas tablas horizontales y verticales, donde la primera de ellas es de tipo horizontal y la segunda de ellas es vertical. La finalidad de estos tipos de tablas es organizar grandes cantidades de información bajo variados factores genéricos. 
En el caso que aparece inmediatamente, cuenta con tres columnas principales con los términos de componentes, modelo y significado. Los datos que aparecen en la primera columna en sus diferentes filas son inamovibles (pero si modificables en función de la investigación) mientras que los demás son parte del ejemplo ya que el diseño simple serían las tres columnas dejando todos los espacios correspondientes a las filas para ser rellenados con los datos recogidos.

- Tablas horizontales y verticales (Miles y Huberman, 1994):

Tabla 4

Ejemplo de tabla horizontal para abreviar resultados para la investigación cualitativa (citado por Codina, 2019)

\begin{tabular}{lll}
\hline Componentes & Modelo & Significado \\
\hline individualización & Estandarización & $\begin{array}{l}\text { Se somete a } \\
\text { individualización } \\
\text { mostrando racionalidad } \\
\text { como respuesta a la } \\
\text { exigencia. }\end{array}$ \\
$\begin{array}{l}\text { Responsabilidad } \\
\text { estudiante }\end{array}$ & \\
Exposición del trabajo & \\
Estrategias de aprendizaje & \\
Entorno familiar & \\
Periodo de tiempo & \\
Selección de estudiantes & \\
Tamaño del programa & \\
\hline
\end{tabular}

Tabla 5

Ejemplo de tabla vertical para abreviar resultados para la investigación cualitativa (citado por Codina, 2019)

\begin{tabular}{lll}
\hline \multicolumn{4}{c}{ Métodos usados } \\
\hline Etiqueta Descripción & Búsqueda Evaluación Síntesis Análisis
\end{tabular}


Al contrario que la primera tabla de ejemplo, ésta cuenta con una columna principal llamada "Métodos usados" y en la fila inferior tenemos los aspectos claves para desarrollar los contenidos extraídos de cualquier investigación. Este punto del artículo presenta nuevamente dos ejemplos de tablas pero con el objetivo concreto de dar respuesta a las siguientes funciones: explorar, describir, ordenar, explicar y predecir.

-Tablas para presentar y relacionar variables (Miles y Huberman, 1994; citado por Codina, 2019):

Tabla 6

Ejemplo de tabla para presentar y relacionar variables

\begin{tabular}{llll}
\hline & \multicolumn{2}{c}{ Efectos directos } & $\begin{array}{l}\text { Tamaño del efecto y } \\
\text { metaevaluación }\end{array}$ \\
\hline CASOS & $\begin{array}{l}\text { Objetivos del Positivos Negativos } \\
\text { programa }\end{array}$ & Positivos Negativos
\end{tabular}

CASO 1

CASO 2

Este modelo de tabla permite presentar los datos, relacionarlos e incluso compararlos con la finalidad de extraer de los resultados más relevantes y menos bajo la segunda fila denominada "positivos y negativos" en cuanto a los efectos directos y la evaluación de los mismos. Es importante clarificar los objetivos para cada caso que se va a presentar.

- Tablas para procesar la información y relacionar variables con las siguientes funciones: explorar, describir, ordenar, explicar y predecir (Miles y Huberman, 1994):

Tabla 7

Ejemplo de tabla para presentar y relacionar variables (citado por Codina, 2019)

\begin{tabular}{ll}
\hline \multicolumn{2}{c}{ CASOS con asistencia prevista } \\
\hline Efectos de la innovación & Sustancial Moderado \\
1. & \\
2. & \\
$3 \ldots$ \\
Efectos individuales \\
1. \\
2. \\
$3 \ldots$ \\
Efectos de la organización \\
\hline
\end{tabular}


1.

2.

$3 \ldots$

Esta Tabla, a diferencia de la anterior, permite representar la información referente a varios casos pero atendiendo a diferentes efectos. Los números indican la cantidad de casos que cuentan con algún tipo de efecto en concreto y el nivel de relevancia teniendo en cuenta la segunda fila "sustancial", "moderado" o "leve".

-Tablas para ubicar cualquier información (Miles y Huberman, 1994; citado por Codina, 2019):

Tabla 8

Ejemplo de tabla para presentar datos

\begin{tabular}{|c|c|c|c|c|c|}
\hline \multirow[t]{2}{*}{$\begin{array}{l}\text { Tipo } \\
\text { resultado }\end{array}$} & de & $\begin{array}{l}\text { Uso temprano } \\
1 \text { y } 2 \text { años }\end{array}$ & & $\begin{array}{l}\text { Uso tardío } \\
3 \text { años }\end{array}$ & \\
\hline & & Efecto directo & $\begin{array}{l}\text { Efecto } \\
\text { indirecto }\end{array}$ & Efecto directo & Efecto indirecto \\
\hline
\end{tabular}

Estructural

Procedimental

Relacional/clima

-Matriz para evaluar el nivel de consenso en el grupo (Onwuegbuzie et al, 2011):

Tabla 9

Evaluación del nivel de consenso en el grupo

\begin{tabular}{llllll}
\hline Pregunta Partic1 Partic2 Partic3 Partic4 Partic5 Partic6 \\
1 \\
2 \\
3 \\
$\ldots \ldots .$.
\end{tabular}


Las siguientes anotaciones podrían ser interesantes en las casillas:

$\mathrm{C}=$ indicación de consenso (verbal o no verbal)

$\mathrm{D}=$ indicación disenso (verbal o no verbal)

$\mathrm{DE}=$ proporciona declaración sustancial o ejemplo que sugieren consenso

$\mathrm{DD}=$ proporciona declaración sustancial o ejemplo que sugieren disenso

$N A=$ no indica consenso o disenso ( $v$. g. no responde)

\section{Metodología}

A lo largo de este artículo se definen los grupos de discusión y grupos focales y a través de este epígrafe se añade que el objetivo propuesto es mostrar el procesamiento y análisis de la información a partir de las entrevistas a estos grupos. Para ello se han utilizado, por una parte los grupos focales con asesores de centros de profesorado (CEPs) que trabajan con redes de centros escolares de la provincia de Sevilla; por otra, grupos de discusión con mentores principiantes pertenecientes a un Programa de inducción profesional docente en República Dominicana. A través de estas entrevistas se procesa la información, se ordena minuciosamente y se elaboran teorías a partir de las propias experiencias con profesionales en ejercicio para la construcción colectiva del conocimiento acerca de un tópico concreto.

Para abordar el análisis de los grupos focales se ha recurrido a un grupo de informantes como estudio de caso formado por 4 asesores más 2 investigadores, siendo su perfil profesional asesores de primaria, secundaria y atención a la diversidad. El tópico tratado ha sido "El asesoramiento a redes educativas de centros escolares" (funcionamiento, impacto, repercusiones, implicaciones, etc.). La entrevista ha sido grabada y desarrollada mediante guiones de trabajo previamente preparados en base a los resultados de investigaciones propias y relacionadas con redes educativas locales desde el año 2000 hasta la actualidad, experiencias de campo y opiniones con entrevistas individuales al profesorado y asesores participantes en estas redes escolares.

La entrevista ha girado en torno al siguiente guión:

- Nivel de participación de los centros en las redes... (opiniones), en qué se traduce esta participación de los centros, características de los centros que participan (mejoras)

- Existencia de un fortalecimiento de dinámicas de trabajo más colaborativas y compartidas por todos a partir de la participación

- Estructura de trabajo de estas REDES, coordinación y funciones de la comunidad educativa de cada centro en relación a profesores/padres/alumnos, normas escritas y asumidas por todos

- Participación en la elaboración de Planes de Mejora de cada centro a partir de la participación en la RED, repercusión en cada centro de las mejoras consensuadas en la red y en el funcionamiento de las prácticas de centro y aula (horario, espacios....)

- Desarrollo de Proyectos inclusivos de centro y de aula, apoyo a los alumnos con necesidades educativas

- Actitudes del profesorado ante procesos formativos propios y en grupo de trabajo... abordaje de los procesos formativos 
- Participación de los distintos sectores de la comunidad educativa (Equipos Directivos, Ayuntamiento, padres, EOE...), porcentaje de participación de cada sector

- Por último: Obstáculos encontrados por las propias Redes para el desarrollo de sus objetivos y obstáculos que encuentran los asesores para trabajar con las Redes

En cuanto a los grupos de discusión, los participantes toman un papel importante en la interacción, diálogo y reflexión de todas las aportaciones nacidas del debate, en respuesta a una serie de cuestiones planteadas. Concretamente, dos grupos de discusión de carácter presencial de 4 y 6 participantes respectivamente. Los objetivos planteados son identificar obstáculos y barreras en el proceso de asesoramiento a docentes principiantes, y explorar la evolución profesional y personal de los mentores para conocer la proyección futura en este desempeño profesional.

Para la selección de las cuestiones se atiende a aspectos concretos como el desarrollo de la mentoría, el asesoramiento y el desarrollo profesional y personal del mentor principiante. La conversación ha girado en torno a la temática de la mentoría en general abordada a partir de dos dimensiones, la mentoría propiamente dicha y el desarrollo profesional y personal. La dimensión "mentoría" abarca aspectos como definición, dificultades, soluciones, ayudas, similitudes y diferencias entre coordinadores pedagógicos y mentores, utilización de la mentoría formal e informal y consejos a futuros mentores principiantes tanto personales como profesionales; la dimensión "desarrollo profesional y personal" explora la formación necesaria para ejercer la figura de "mentor", cualidades del mentor principiante, disponibilidad de tiempo (otros cargos laborales) e innovación en los modos de acción de la labor de mentoría.

Se aborda el análisis de la información de estos grupos mediante el diseño de matrices y tablas generadas a partir de un proceso interpretativo de tipo categorial. Se trata de un análisis del discurso "que implica seleccionar segmentos representativos o excepcionales y luego analizarlos en detalle" (Onwuegbuzie et al, 2011, p. 138).

El procedimiento del análisis de los datos se ha fundamentado en la secuencia de Saldanha da Silveira, Colomé, Heck, Nunes da Silva y Viero (2015):

1. Fase pre-análisis: organización del material de los datos obtenidos en las grabaciones del grupo y transcripción para construir el corpus de la investigación y construcción de las categorías a partir del análisis temático del pre-análisis utilizando en el análisis de contenido siempre un orden semántico. A continuación, construcción de la tabla/matriz con los siguientes elementos: participantes, tema de discusión, contribuciones de las personas sobre el tema, reflexiones de investigadores, observaciones, etc. Los elementos para incluir en las tablas/matrices deben ser pensados en función de los objetivos de la investigación.

2. Fase de exploración del material: a partir de la organización del material, se procede al diseño de tablas/matrices, dada la gran cantidad de datos extraídos de las sesiones, en función de los datos que deben ser depurados y organizados en relación a la temática de estudio de modo que los datos de los tablas/matrices se organicen en módulos y temas

3. Fase de tratamiento de los resultados: diseño de modelos de tablas y matrices adecuados para el análisis de los datos obtenidos. Con estas matrices el investigador puede reflexionar de manera sistemática en el análisis de los resultados, compararlos y construir las ideas compartidas a partir de estos. 


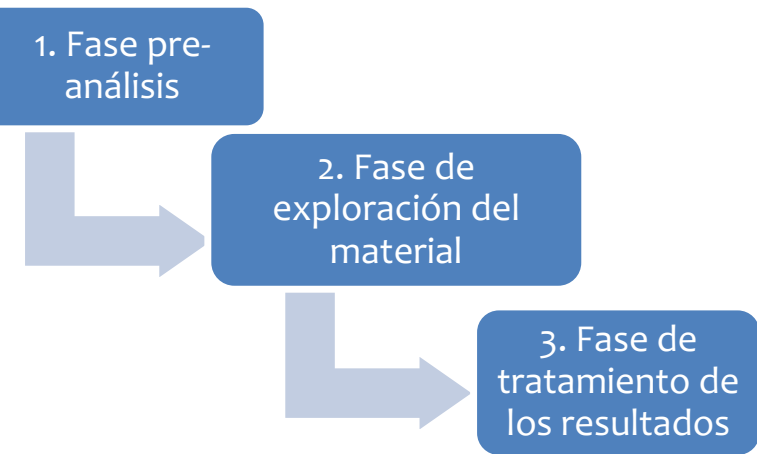

Figura 1. Procedimiento de análisis de los datos, basado en Saldanha et al (2015)

Se presentan a continuación dos modelos para el procedimiento de extracción de categorías de análisis.

El proceso para la elaboración conceptual de la información de modo organizado y coherente de los resultados de la entrevista en grupos focales, y construir conocimiento a partir de esta técnica, se basa en una matriz de cuatro entradas de categorías y subcategorías para estructurar la información en base a criterios temáticos. Se trata de datos que reflejan la comprensión de los procesos y las situaciones por parte de los propios participantes en los contextos de redes estudiados. El procedimiento de reducción de los datos ha sido: división de la información según criterios temáticos, identificación de categorías y codificación descriptiva de la información (estrategias de selección secuencial) y a partir de aquí se construye la matriz cualitativa.

Tabla 10

Grupo Focal X: asesores de centros de profesores (CEPs).

Grupo Focal X: asesores de centros de profesores (CEPs)

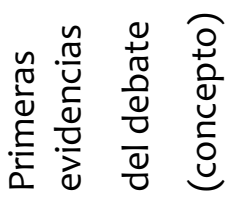

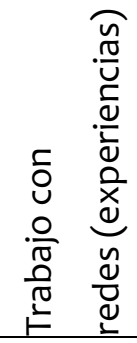



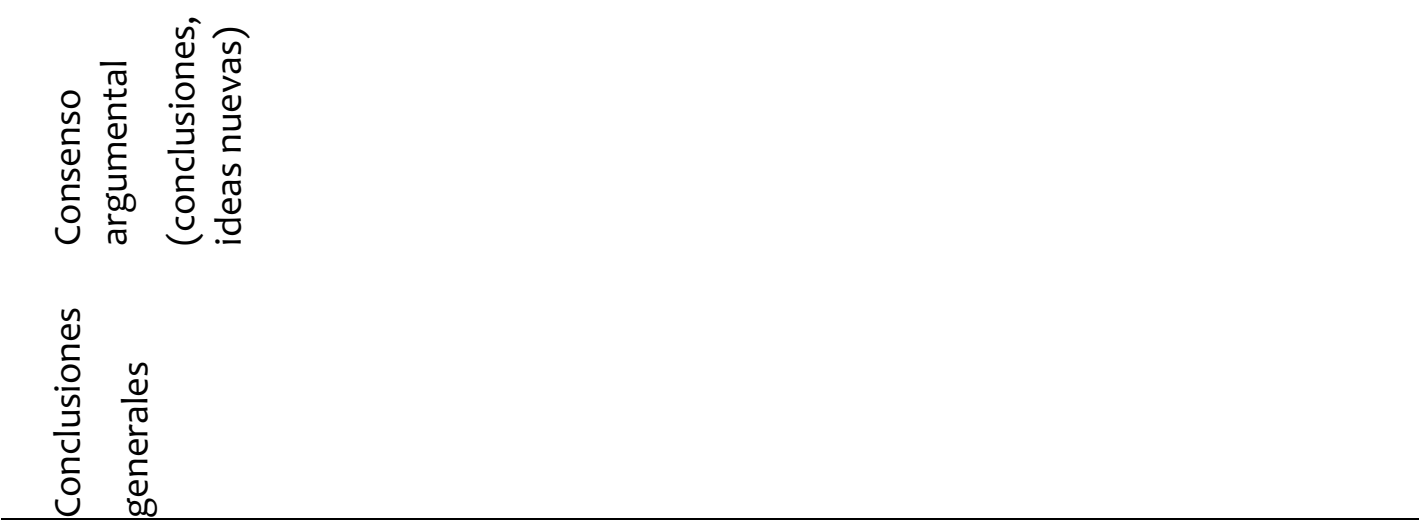

Nota: Elaboración propia.

Las categorías y subcategorías son las siguientes:

CATEGORÍA A. Primeras evidencias del debate: escena comunicativa y tópico a desarrollar

Subcategorías: planificación, tiempo, contenidos, evaluación, metodología, resolución de inquietudes, acompañamiento profesional y retroalimentación

CATEGORÍA B. Trabajo con redes: construcción del concepto y experiencias concretas del trabajo en red

Subcategorías: prácticas innovadoras, trabajo colaborativo, equipo de trabajo, transversalidad, buenas prácticas, participación familias

CATEGoRíA C. Consenso argumental: acuerdos consensuados del trabajo en redes (conclusiones, nuevas ideas...)

Subcategorías: atención a necesidades del sistema, desarrollo de Proyectos inclusivos, figura dinamizadores de red (dinamizadores "silentes")

CATEGORÍA D. Conclusiones generales de ideas: puesta en común

Subcategorías: motivación, cohesión grupal, fortalecimiento de relaciones, oportunidades de aprendizaje, prevención aislamiento profesional, implicación familias, mejora aprendizaje del alumnado

A partir de estos criterios temáticos se examinan las valoraciones de los participantes acerca del trabajo de asesoramiento en los centros escolares. Como ejemplo de ello se muestran las categorías de análisis para los grupos focales a través de un gráfico en red circular realizado con el programa informático Atlas. Ti 8:

Tal y como se observa en el Gráfico 1 se dispone de cuatro categorías principales, anteriormente detalladas. Éstas cuentan con diversas categorías sombreadas en diferentes colores. Las amarillas se enlazan con la categoría de trabajo con Redes; las rojas con las primeras evidencias debate; las grises con el consenso argumental y las azules con la categoría de conclusiones. 


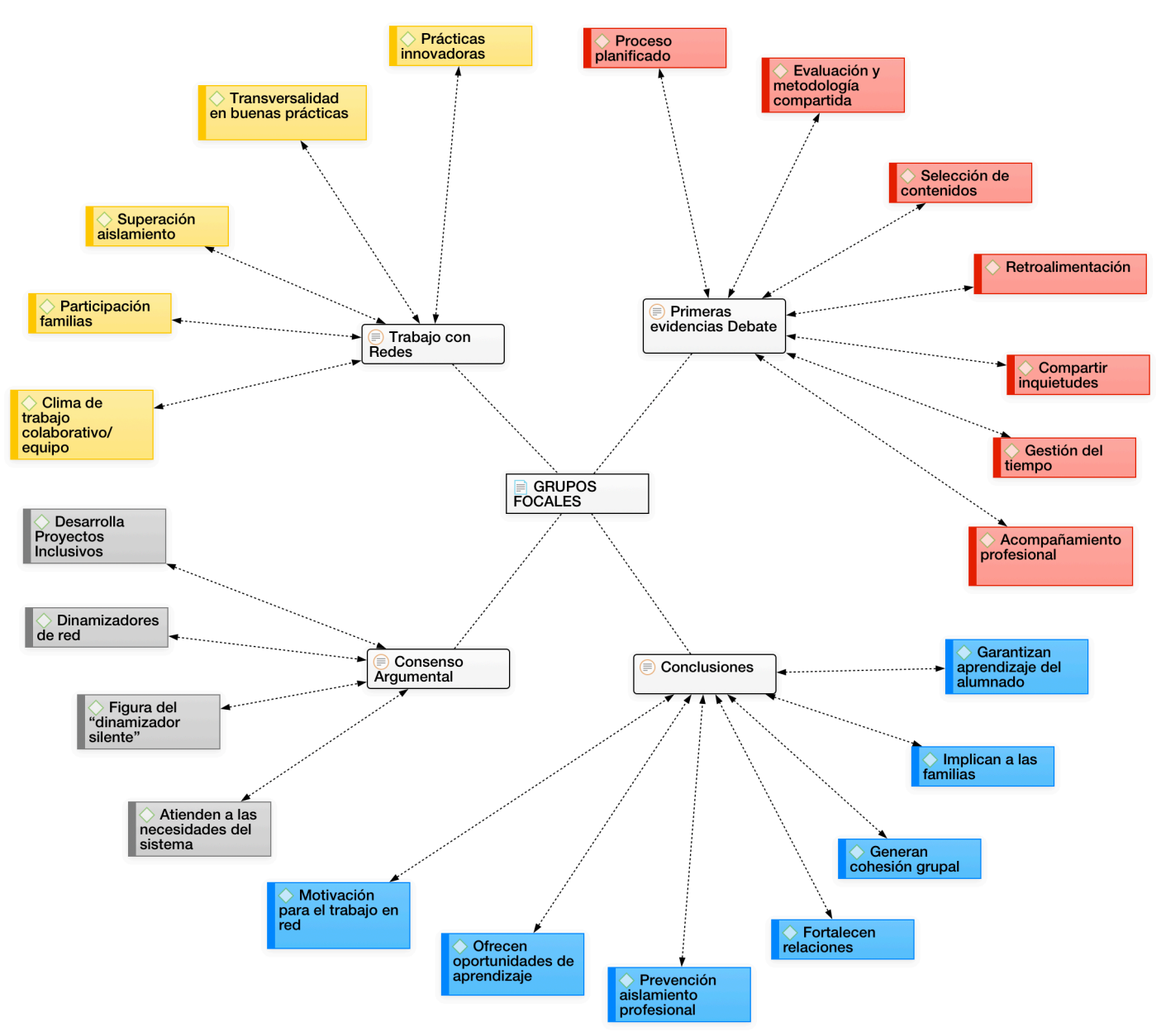

Gráfico 1. Categorías de análisis para los grupos focales. Fuente: elaboración propia.

Como ejemplo de la segunda matriz, en este caso horizontal, para los grupos de discusión se aporta la siguiente Tabla (Tabla 11).

Tabla 11

Grupos de discusión.

\begin{tabular}{|c|c|c|c|c|c|}
\hline Dimensiones & Categorías & Objetivo & $\begin{array}{l}\text { Grupo } \\
\text { discusión } \\
1\end{array}$ & $\begin{array}{l}\text { Grupo } \\
\text { discusión } \\
2\end{array}$ & $\begin{array}{l}\text { Análisis } \\
\text { comparativo } 1 \\
\text { y } 2\end{array}$ \\
\hline
\end{tabular}

Nota: Elaboración propia.

En esta segunda matriz perteneciente a grupos de discusión, se realiza un análisis detallado en función de las dimensiones y categorías diseñadas para cada grupo por separado y el análisis comparativo entre ambos. Este modelo (ver Tabla 12) ofrece una Tabla de seis entradas horizontales que se pueden reducir a cuatro (dimensiones, categorías, objetivos y grupo discusión). En este estudio aparecen dos grupos de discusión, presentando el sistema 
de matrices de tal forma que la primera columna "dimensiones" es general e indica la temática o temáticas principales a investigar; la segunda columna "categorías" es específica, trata cuestiones a plantear; la tercera columna trata el "objetivo" de la cuestión planteada, lo que se quiere indagar; la cuarta columna alude a las respuestas del primer grupo de discusión; la quinta columna se refiere a las respuestas referentes al segundo grupo de discusión y la última columna permite avanzar en un análisis comparativo entre ambos grupos de discusión sin olvidar las dimensiones y categorías principales de la investigación.

El procedimiento para la extracción de categorías de análisis se realiza con dimensiones, las cuales cuentan con diversas categorías comunes para ambos grupos de discusión. La recogida de los datos es predominantemente visual y da acceso directo a la información para su posterior análisis. Para ello hemos utilizado el programa informático Atlas. Ti versión 8 , en el cual, se ha incorporado la transcripción de ambos grupos, creando dimensiones, categorías y códigos.

A continuación se muestra un ejemplo representativo de una gráfica en red en forma cuadrada basada en la matriz para grupos de discusión:

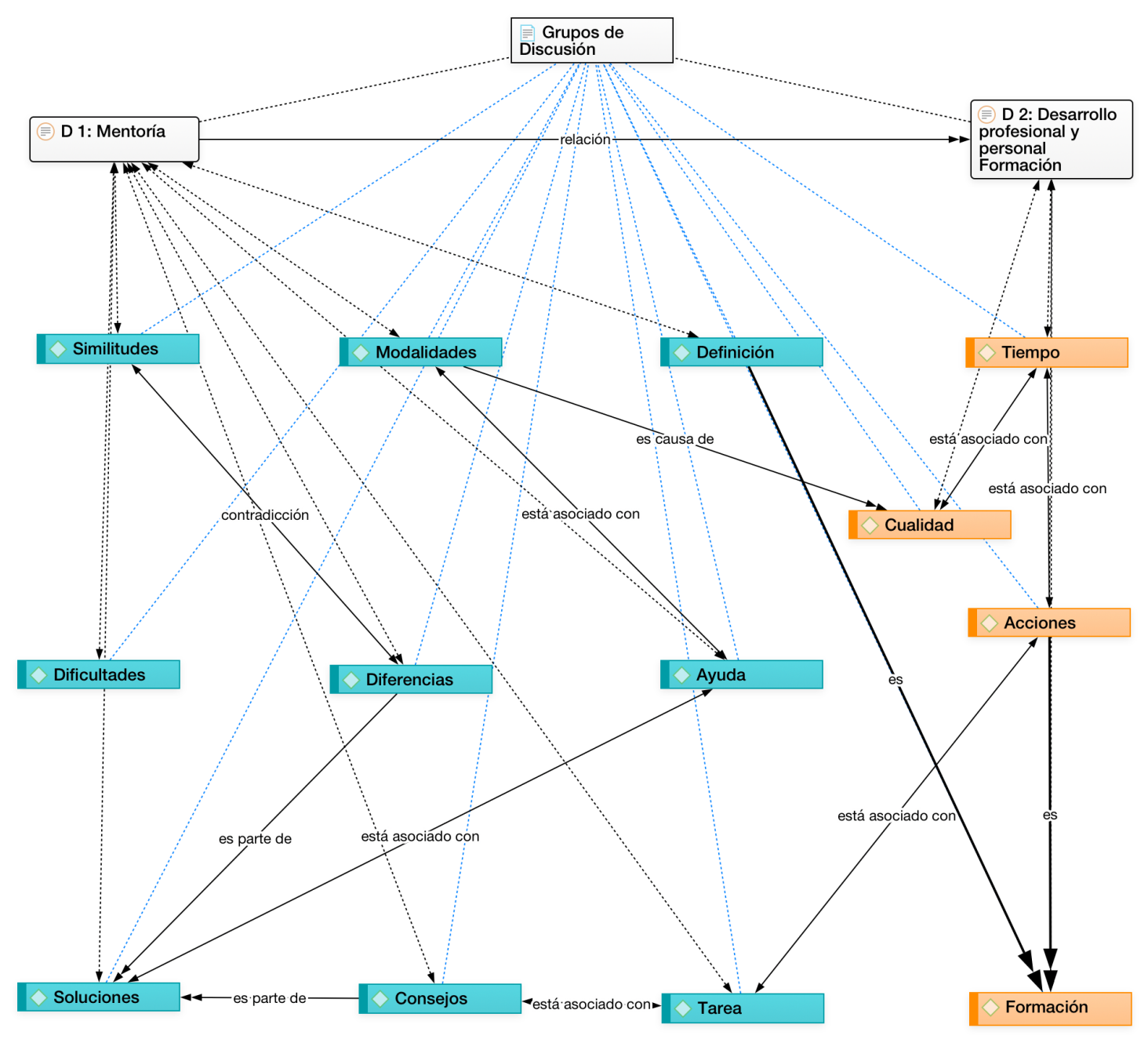

Gráfico 2. Categorías de análisis para los grupos de discusión. Fuente: elaboración propia.

El gráfico en red (Gráfico 2), a través de las dimensiones y categorías de análisis, permite realizar comparaciones para resaltar asociaciones, semejanzas y diferencias entre los 
diferentes grupos de resultados. Las categorías asignadas para la dimensión Mentoría son las sombreadas en azul mientras que las categorías para la dimensión de Desarrollo Profesional y Personal-Formación están sombreadas en naranja. Una vez cumplimentado el sistema de matrices se pasa a profundizar en los resultados obtenidos. De igual modo, se pueden colocar aportaciones individuales relevantes para su análisis posterior. En los ejemplos mostrados anteriormente se pueden modificar las columnas y filas en función del interés del estudio.

\section{Discusión y conclusiones}

En estas conclusiones se extraen algunas indicaciones emergentes del trabajo con estas herramientas de análisis para la investigación basada en la reflexión colectiva. La experiencia en estos estudios indica que el análisis de la información debe realizarse de forma organizada y ordenada para que los conocimientos que aportan estos profesionales sean de utilidad e interés, aportando sugerencias que parten de su propia experiencia y se generan a partir del grupo. Es primordial utilizar un sistema de análisis para llegar a acuerdos en el propio desarrollo de la conversación grupal acerca del tópico de estudio.

Las conclusiones que se extraen del análisis de los datos en tablas y matrices se concretan en las siguientes:

- El tratamiento de la información ordenada en tablas y matrices genera motivación en el investigador y los propios participantes; además, el análisis colectivo de los temas entre profesionales y su visualización lleva a conclusiones compartidas y participativas que generan conocimiento y compromiso profesional (López, Navarro y Hernández, 2016; Hernández y Navarro, 2017, 2018)

- La participación en el grupo a partir de un planteamiento horizontal, en igualdad y comunicativo entre los miembros genera asimismo confianza entre los participantes (García Calvente y Mateo, 2000)

- La aportación de los participantes, si se realiza de forma visual in situ, implementa formas de trabajo consensuadas a partir de cada una de las contribuciones al grupo (Hamui-Sutton y Varela-Ruiz, 2013)

- El registro de las intervenciones y posterior puesta en común incrementa el intercambio de actitudes formativas hacia procesos de mejora e innovación, por ejemplo, para la evaluación de programas, aspectos curriculares y didácticos, planificación, evaluación educativa, etc. (Hamui-Sutton y Varela-Ruiz, 2013).

- La utilización de tablas y matrices implica un escrutinio para el investigador a partir del análisis de todo el conjunto de datos y la adopción de criterios de investigación cualitativa, elaborando ejemplos y secuencias a partir de los datos (Markee, 2000)

- El examen del «cómo» y el «qué» de las interacciones de los participantes y las interacciones entre los mismos produce una mayor riqueza en los datos (Onwuegbuzie et al, 2011).

- El estudio del consenso en los grupos favorece la formación de teorías estables a partir del análisis de la información entre prácticas docentes y entre "colegas", evitando el aislamiento profesional y "creando estructuras faciliten y promuevan la colaboración docente" (Bolívar, 2015, p.2).

- El registro en tablas y matrices genera control y gestión de los tiempos en las exposiciones desde los propios participantes, evitando el uso excesivo del mismo. 
Como señalan García-Carretero, Codina y Pedraza-Jiménez (2016), “corresponde a cada investigador, en función de sus intereses y de los parámetros de su proyecto, decidir qué indicadores utilizará" (p.30), incluso si selecciona únicamente una sola tabla o combina varias de ellas. Representar la información cualitativa se convierte en calidad debido a la responsabilidad de los autores por presentar los datos de forma clara y concisa destacando los conceptos (Codina, 2019).

Este tipo de estudios permite ahondar y comprender la complejidad que comporta la escucha activa compartida para la construcción del conocimiento y procesarla para su posterior interpretación y puesta en común, siendo este su valor principal. Poner en práctica estrategias grupales y su análisis, contribuye a tomar conciencia de este potencial, permitiendo, a través de tablas y matrices, la visualización de la construcción de aprendizajes significativos.

Como señala Codina (2019), ningún trabajo académico de tipo cualitativo debería presentarse sin un buen número de tablas, ya que portan claridad y la transparencia a su trabajo, síntesis de resultados e ideas, aumentan la calidad, favorecen la comprensión y ayudan a visualizar la información de la investigación.

\section{Referencias}

Barbour, R. S., Amo, T., y Blanco, C. (2013). Los grupos de discusión en investigación cualitativa. Madrid: Morata.

Bolívar, A. (2015). Construir localmente la capacidad de mejora: Liderazgo pedagógico y Comunidad Profesional. Comunicación presentada al XIII Congreso Nacional de Investigación Educativa. Chihuahua (México), noviembre.

Codina, L. (2019). Sintetizar y representar información cualitativa: tablas y diagramas en trabajos de final de máster y tesis doctorales. Recuperado el 25 de noviembre de 2019 de: https://www.lluiscodina.com/tablas-diagramas-investigacion-cualitativa/

Gutiérrez Brito, J. (2009). Técnicas grupales. En J. Callejo (coord.). Introducción a las técnicas de investigación social (pp. 95-115). Madrid: Ramón Areces.

García-Carretero, L., Codina, L., y Pedraza-Jiménez, R. (2016). Indicadores para el estudio de la visibilidad y del impacto de los cibermedios en el ecosistema digital. Barcelona: Editorial Digidoc.

García Calvente, M., y Mateo, I. (2000). El grupo focal como técnica de investigación cualitativa en salud: diseño y puesta en práctica. Atención Primaria, 25(3), 181-186.

Hamui-Sutton, A., y Varela-Ruiz, M. (2013). La técnica de los grupos focales. Investigación en Educación Médica, 2(1), 55-60.

Hernández, E., y Navarro, M.J. (2017). Las redes educativas para trabajar mejor desarrollando procesos de aprendizaje. Revista Electrónica de Investigación Educativa, 20 (3), 29 -42.

Hernández, E., y Navarro, M.J. (2018). La participación en redes escolares locales para promover la mejora educativa, un estudio de caso. Profesorado, 22 (2), 49-68.

Ibarrola-García, S. (2012). La convivencia escolar en positivo. Madrid: Pirámide

Markee, N. (2000). Conversation analysis. Mahwah, NJ, EE. UU.: Lawrence Erlbaum. 
Miles, M., y Huberman, A. (1994). Qualitative data analysis: an expanded sourcedbook. Thousand Oaks: Sage Pubications.

Miles, M., Huberman, A., y Saldaña, J. (2014). Qualitative Data Analysis. A Methods Sourcebook. London: Sage Publications.

Navarro, M.J., y Hernández, E. (2017). La colaboración en red entre profesorado de aulas específicas de autismo para promover el intercambio profesional para la inclusión educativa. Perfiles Educativos, 39 (156), 58-71.

Onwuegbuzie, A. J., Dickinson, W. B., Leech, N. L., y Zoran, A. G. (2011). Un marco cualitativo para la recolección y análisis de datos en la investigación basada en grupos focales. Paradigmas: Una Revista Disciplinar de Investigación, 3 (1), 127-157.

Saldanha da Silveira, D., Donaduzzi, Colomé, C., Teresinha Heck, T., Nunes da Silva, M., y Viviani, F. (2015). Grupo focal y análisis de contenido en investigación cualitativa. Index de Enfermería, 24 (1-2), 71-75.

Thofehrn, M. B., Montesinos, M. J., Porto, A. R., Amestoy, S. C., Arriera, I. C., y Mikla, M. (2013). Grupo focal: Una técnica de recogida de datos en investigaciones cualitativas. Index de Enfermería, 22 (1-2), 75-78.

Vivar, C., Arantzamendi, M., López-Dicastillo, O. y Gordo, C. (2010). La Teoría Fundamentada como Metodología de Investigación Cualitativa en Enfermería. Index Enfermería, 19 (4), 283-288.

Wilkinson, S. (2004). Focus Group research. En D. Silverman (ed.), Qualitative Research: Theory, method and practice (pp. 177-199). Thousand Oaks: Sage.

Wilkinson, S. (2012). Focus group methodology: A review. En G. Walden (ed), Focus Group Research (p. 181). London: Sage. 